\title{
Flow Behavior of Herschel-Bulkley Fluid in a Slot Die
}

\author{
Masayuki NAGASHIMA*, Tomiichi HASEGAWA ${ }^{* *}$, and Takatsune NARUMI ${ }^{* *}$ \\ *Dai Nippon Printing Co., Ltd. 1-3, Midorigahara, 1-chome, Tsukuba-shi, Ibaraki 300-2646, Japan \\ ${ }^{* *}$ Faculty of Engineering, Niigata University 8050, 2-no-cho, Ikarashi, Niigata 950-2181, Japan
}

(Received : April 25, 2006)

\begin{abstract}
To achieve uniform thickness of coated films using non-Newtonian fluids, we are often required well-designed geometries of flow channel in a slot die. This is the case especially for fluids with the properties of both shear thinning and yield stress which is non-negligible in a range of low shear rate such as in an intermittent die coating. In the present work, flow in the slot die has been studied using Herschel-Bulkley fluid expressing both shear thinning and yield stress. The Herschel-Bulkley fluid includes power-law and Bingham fluid models comprehensively. This fluid model is applicable to many real fluids. We derive simplified equations for the outflow distribution as functions of power-law index, yield stress and the geometry parameters of the die. Furthermore, we propose a useful designing method for predicting the optimum geometry so as to guarantee uniformity of outflow from the die slot, based on the HerschelBulkley fluid. The value of this method is confirmed by comparing it with experimental results and with previous theoretical results for the power-law and Bingham fluid models.
\end{abstract}

Key Words: Herschel-Bulkley fluid / Shear thinning / Yield stress / Flow behavior / Algebraic analysis

\section{INTRODUCTION}

A slot die has been used in a manufacturing process to form a two-dimensional film, and is especially useful when high uniformity of the film thickness is required. This slot-die coating is most suitable for processing in the fields of electronics and industrial materials, because the coating can expend only a necessary amount of costly liquids pre-metered for coating. Furthermore, the liquids are less contaminated by this method, due to its air-closed system.

The flow channel of a typical slot die essentially consists of a cavity and a slot. The cavity is a distribution chamber used to redistribute fluids from the inlet to the end of the chamber. The slot is a paper-thin slit to supply fluids to a substrate so as to form a liquid film.

When uniform film thickness is required, it is necessary to suppress deviation of the slot flow from uniformity. It is well known that Newtonian fluids flow with a little deviation even if the cavity and the slot have constant geometries along the flow direction, and that some non-Newtonian fluids having non-dilatancy ${ }^{1)}$ display large deviations. ${ }^{2-4)}$

There are many kinds of sophisticated material to be coated in modern engineering such as magnetic powders, electrosensitive micro capsules, etc. They are dispersed in a liquid as colloidal dispersions or suspensions and are coated on a substrate. At that time the liquid often reveals non-

\section{Newtonian behavior. ${ }^{5-7)}$}

We can control the outflow from a slot to achieve uniformity by exploiting and designing the slot die. ${ }^{8,9)}$ However, designing methods based on trial-and-error using threedimensional numerical analysis ${ }^{9-12)}$ such as FEM or FVM take a large amount of time. This is due to the millions of mesh that is needed. Consequently, engineers have developed simple designing systems based on simplified flow equations for the flows inside the die.

Weinstein et al. have proposed efficient and precise designing methods using solutions of approximate equations for several non-Newtonian fluid models having shear thinning, such as power-law fluid, truncated-power-law fluid and Ellis fluid. ${ }^{8}{ }^{13-17)}$ The apparent viscosities of truncated-power-law fluid and Ellis fluid approach constant values as the shear rate decreases to zero, whereas one of power-law fluid increases.

However, there exist some extraordinary fluids which increase their apparent viscosities more than power-law fluid with decreasing shear rate. They are found particularly in concentrated suspensions, with dispersed particles on the order of microns, and colloidal dispersions and emulsions.

When the velocity inside the die is low, as in an intermittent die coating, the flow of these fluids increases the deviation of the film thickness from uniformity. Therefore, it is useful to adopt a fluid model that provides the yield stress in analyzing the flows of such fluids. Some arguments have been presented 
whether the yield stress practically exists or not and several reports describe that these fluids show Newtonian feature even when the shear rate is close to zero. Papanastasiou et al. have given a model expressing these phenomena. ${ }^{18,19)}$ However, there is no problem in assuming the existence of yield stress in the die flow usually used in industry.

In a previous paper, the influence of yield stress on the die flow $^{20)}$ was reported. It was also proposed that a flow distribution model and an efficient designing method for seeking the optimum geometry corresponding to a flow distribution inside the die should be used in the case of Bingham fluid. This method is useful only when the difference between shear stress and yield stress is linear against shear rate.

Herschel-Bulkley fluid, expressing both shear thinning and yield stress, is in common use, chiefly because it fuses the power-law fluid model and Bingham fluid model. HerschelBulkley fluid model is available for many practical fluids, but the studies of flow inside die using this fluid model have hardly been found.

In the present study, we propose an efficient method for designing the optimum geometry to attain the smallest deviation of the slot outflow from uniformity using a Herschel-Bulkley fluid. There should be agreement between the analytical results and experimental data obtained by the authors and the results of previous studies ${ }^{8,20)}$ for power-law fluid and Bingham fluid.

\section{NOMENCLATURE}

\section{$B$ : Half of a slot gap at $x^{*}=1$}

$B\left(x^{*}\right)$ : Half of a slot gap at given $x^{*}$

$L$ : Slot length at $x^{*}=1$

$L\left(x^{*}\right)$ : Slot length at given $x^{*}$

$N_{\mathrm{HB}}:$ Generalized Bingham number $=\tau_{0} R^{\mathrm{n}} /\left(\mu_{0} \bar{v}_{0}{ }^{\mathrm{n}}\right)$

$n$ : Power-law index

$p$ : Pressure in a die

$p\left(x^{*}\right)$ : Pressure in a cavity

$p^{*}\left(x^{*}\right)$ : Dimensionless pressure $=p\left(x^{*}\right) R^{\mathrm{n}} /\left(\mu_{0} \bar{v}_{0}{ }^{\mathrm{n}}\right)$

$q_{z}$ : Flow rate of a slot per unit die width

$q_{z}^{*}$ : Normalized flow rate of a slot per unit die width

$Q_{0}$ : Flow rate through a cavity at $x^{*}=0$ (=influx)

$Q_{\mathrm{x}}$ : Flow rate through a cavity

$Q_{\mathrm{x}}{ }^{*}$ : Normalized flow rate through a cavity

$R$ : Radius of cross section of a cavity

$v$ : Velocity in a die

$v_{x}: x$-component of the velocity in a cavity

$\bar{v}_{0}$ : Average $x$-axial velocity in a cavity at $x^{*}=0$
$W:$ Die width

$\dot{\gamma}$ : Shear rate

$\mu_{0}$ : Apparent viscosity at a reference shear rate

$\tau_{0}:$ Yield stress

$\tau:$ Stress tensor for fluids

*: Refers to the dimensionless variables

\section{MODEL FOR FLOW ANALYSIS}

\subsection{Geometry Inside the Die}

Figure 1 depicts a typical slot die consisting of a cavity and a slot. ${ }^{20)}$ The cavity distributes a liquid from the inlet to the end of width $W$. The liquid is then extruded through the slot to form a liquid film on a substrate. The cross section of the cavity is a circle with a constant radius $R$.

The longitudinal geometry of the cavity is a cylinder, and the slot resembles a rectangular channel bearing a cross section of large aspect ratio. We therefore adopt the cylindrical coordinate system $(r, \theta, x)$ for the cavity and Cartesian coordinates $(x, y, z)$ for the slot. For the cavity, $x=0$ and $x=W$ refer to the inlet and the end of the cavity, respectively. $x$ is normalized as $x^{*}=x / W$ and $r$ is expressed as $r^{*}=r / R$.

For the slot, the $x$-axis is taken in common with the coordinate of the cavity. The $y$-axis shows the direction normal to the flow direction, $y=0$ being the centre plane between the slot walls; the $z$-axis coincides with the flow direction, $z=0$ being the slot inlet connected to the cavity.

The cross-section of the slot in the $x$-direction is a thin rectangle. The slot gap is written as $2 B(x)$ and the slot length, meaning the distance between the slot inlet and outlet, is $L(x)$. Variations of $B(x)$ and $L(x)$ are usually very small (less than $0.1 \%$ and $1 \%$, respectively). Here $y$ and $z$ are normalized as $y^{*}=y / B$ and as $z^{*}=z / L$.

We call the prerequisite parameters needed in designing a geometry $(W, R, L, B)$ the geometry setting parameters.

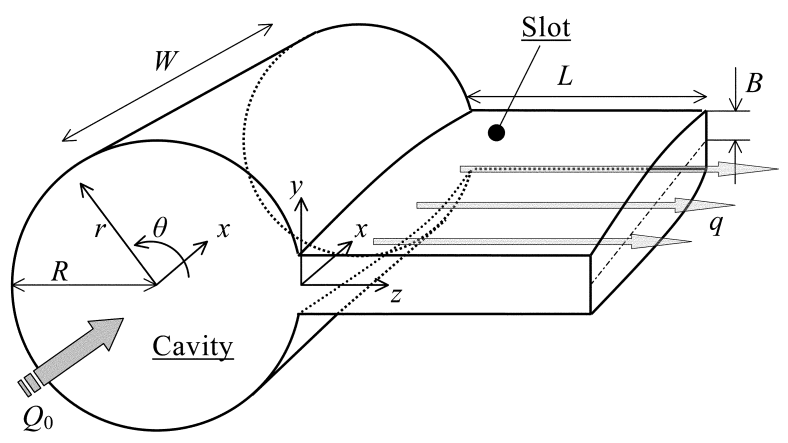

Fig. 1. Geometry of cavity dies. 
Fluids flowing through both the cavity and the slot are described by the following momentum and mass balance equations in each coordination system under isothermal, steady and incompressible conditions. In this case, the inertia term and the body force term are neglected ${ }^{21)}$ because of the superiority of the yield stress and the viscous stress.

$$
\begin{aligned}
& -\nabla p+\nabla \cdot \boldsymbol{\tau}=\mathbf{0} \\
& \nabla \cdot \mathbf{v}=0
\end{aligned}
$$

\subsection{Formulation of Flow Inside the Die}

\subsubsection{Flow in the cavity}

In the cavity flow, it is assumed that the velocity component toward the slot in the cavity is negligible and only the $x$-component in the governing equation of momentum remains. This assumption was justified by Weinstein et al. ${ }^{14,16)}$ and has been used widely. ${ }^{1,4,13-17)}$

Using Herschel-Bulkley model for $\tau$ in Eq. (1) and normalizing it, we have

$$
-\frac{1}{W} \frac{d p}{d x^{*}}=\frac{1}{R r^{*}} \frac{d}{d r^{*}}\left(r^{*}\left(\frac{\mu_{0}}{R^{n}}\left(-\frac{d v_{x}}{d r^{*}}\right)^{n}+\tau_{0}\right)\right)
$$

The velocity is obtained by solving this equation, where $p\left(x^{*}\right)$ is the pressure in the cavity as a function of $x^{*}$, that is,

$$
\begin{gathered}
\left.v_{x}\right|_{\left|\tau_{r x}\right| \geq \tau_{0}}=\frac{n}{n+1}\left(\frac{-1}{2 \mu_{0} W} \frac{d p\left(x^{*}\right)}{d x^{*}}\right)^{\frac{1}{n}} \\
\left(\left(R+2 \tau_{0} W\left(\frac{d p\left(x^{*}\right)}{d x^{*}}\right)^{-1}\right)^{\frac{n+1}{n}}-\left(r+2 \tau_{0} W\left(\frac{d p\left(x^{*}\right)}{d x^{*}}\right)^{-1}\right)^{\frac{n+1}{n}}\right)\left|\tau_{r x}\right| \geq \tau_{0} \\
\left.v_{x}\right|_{\left|\tau_{r x}\right| \leq \tau_{0}}=\frac{n}{n+1}\left(\frac{-1}{2 \mu_{0} W} \frac{d p\left(x^{*}\right)}{d x^{*}}\right)^{\frac{1}{n}} \\
\left(R+2 \tau_{0} W\left(\frac{d p\left(x^{*}\right)}{d x^{*}}\right)^{-1}\right)^{\frac{n+1}{n}}\left|\tau_{r x}\right| \leq \tau_{0}
\end{gathered}
$$

The flow rate through the cavity $Q_{x}$ is given as a function of $d p\left(x^{*}\right) / d x^{*}$ by integrating the velocity in the $x$-direction.

$$
\begin{aligned}
Q_{x}= & \frac{\pi n}{(n+1)(2 n+1)(3 n+1)}\left(\frac{-1}{2 \mu_{0} W} \frac{d p\left(x^{*}\right)}{d x^{*}}\right)^{\frac{1}{n}}\left(R+2 \tau_{0} W\left(\frac{d p\left(x^{*}\right)}{d x^{*}}\right)^{-1}\right)^{\frac{n+1}{n}} \\
& \left\{(n+1)(2 n+1) R^{2}-4 n(n+1) \tau_{0} W R\left(\frac{d p\left(x^{*}\right)}{d x^{*}}\right)^{-1}+8 n^{2} \tau_{0}^{2} W^{2}\left(\frac{d p\left(x^{*}\right)}{d x^{*}}\right)^{-2}\right\}
\end{aligned}
$$

\subsubsection{Flow in the slot}

It is assumed that the pressure gradient in the $x$-direction of the slot is negligible compared with that in the $z$-direction, and that only the $z$-component of Eq. (1) remains as the governing equation. ${ }^{14,16)}$

After normalizing the coordinates and adopting the Herschel-Bulkley model in Eq. (1), we have

$$
-\frac{1}{L} \frac{d p}{d z^{*}}=\frac{1}{B} \frac{d}{d y^{*}}\left(\frac{\mu_{0}}{B^{n}}\left(-\frac{d v_{z}}{d y^{*}}\right)^{n}+\tau_{0}\right)
$$

Then the velocity in the slot is derived by this equation, where $d p / d z^{*}=p\left(x^{*}\right) /\left(L\left(x^{*}\right) / L\right)$, and we obtain

$$
\begin{aligned}
& \left.v_{z}\right|_{\left|\tau_{y z}\right| \geq \tau_{0}}=\frac{n}{n+1}\left(\frac{1}{\mu_{0}} \frac{p\left(x^{*}\right)}{L\left(x^{*}\right)}\right)^{\frac{1}{n}} \\
& \left(\left(B\left(x^{*}\right)-\tau_{0} \frac{L\left(x^{*}\right)}{p\left(x^{*}\right)}\right)^{\frac{n+1}{n}}-\left(B y^{*}-\tau_{0} \frac{L\left(x^{*}\right)}{p\left(x^{*}\right)}\right)^{\frac{n+1}{n}}\right) \\
& \left|\tau_{y z}\right| \geq \tau_{0} \\
& \left.v_{z}\right|_{\left|\tau_{y z}\right| \leq \tau_{0}}=\frac{n}{n+1}\left(\frac{1}{\mu_{0}} \frac{p\left(x^{*}\right)}{L\left(x^{*}\right)}\right)^{\frac{1}{n}}\left(\left(B\left(x^{*}\right)-\tau_{0} \frac{L\left(x^{*}\right)}{p\left(x^{*}\right)}\right)^{\frac{n+1}{n}}\right) \\
& \left|\tau_{y z}\right| \leq \tau_{0}
\end{aligned}
$$

The flow rate of a slot per unit die width $q_{z}$ is determined as a function of $p\left(x^{*}\right)$ and $B\left(x^{*}\right)$ by integrating the velocity in the $z$-direction.

$$
\begin{aligned}
q_{z}= & \frac{2 n}{(n+1)(2 n+1)}\left(\frac{1}{\mu_{0}} \frac{p\left(x^{*}\right)}{L\left(x^{*}\right)}\right)^{\frac{1}{n}}\left(B\left(x^{*}\right)-\tau_{0} \frac{L\left(x^{*}\right)}{p\left(x^{*}\right)}\right)^{\frac{n+1}{n}} \\
& \left((n+1) B\left(x^{*}\right)+n \tau_{0} \frac{L\left(x^{*}\right)}{p\left(x^{*}\right)}\right)
\end{aligned}
$$

We now use the following dimensionless parameters in these equations.

$$
\begin{aligned}
& N_{\mathrm{HB}}=\frac{\tau_{0} R^{n}}{\mu_{0} \bar{v}_{0}{ }^{n}} \\
& p^{*}\left(x^{*}\right)=\frac{R^{n}}{\mu_{0} \bar{v}_{0}{ }^{n}} p\left(x^{*}\right)
\end{aligned}
$$

where $N_{\mathrm{HB}}$ denotes the ratio of yield stress to viscous stress in the cavity, and is referred to as the generalized Bingham number (it becomes the Bingham number ${ }^{22)}$ when $n=1$.). $p^{*}\left(x^{*}\right)$ 
is the ratio of pressure to viscous stress in the cavity. An average velocity at $x^{*}=0$ is defined as $\bar{v}_{0}$.

The flow rate through the cavity is, after normalization using these parameters,

$$
\begin{aligned}
& Q_{x}^{*}=\frac{n}{(n+1)(2 n+1)(3 n+1)}\left(-\frac{1}{2} \frac{R}{W} p^{* \prime}\left(x^{*}\right)\right)^{\frac{1}{n}}\left(1+2 \frac{W}{R} \frac{N_{\mathrm{HB}}}{p^{* \prime}\left(x^{*}\right)}\right)^{\frac{n+1}{n}} \\
& \left((n+1)(2 n+1)-4 n(n+1)\left(\frac{W}{R} \frac{N_{\mathrm{HB}}}{p^{* \prime}\left(x^{*}\right)}\right)+8 n^{2}\left(\frac{W}{R} \frac{N_{\mathrm{HB}}}{p^{* \prime}\left(x^{*}\right)}\right)^{2}\right)
\end{aligned}
$$

where $p^{* \prime}\left(x^{*}\right)=d p^{*}\left(x^{*}\right) / d x^{*} \cdot Q_{x}^{*}$ is unity at $x^{*}=0$ and zero at $x^{*}=1 .\left(0 \leq Q_{x}^{*} \leq 1\right)$

Similarly, flow rate of the slot per unit die width is, after normalization using the parameters in Eq. (10),

$$
\begin{aligned}
q_{z}^{*}= & \frac{2 n}{(n+1)(2 n+1)} \frac{B\left(x^{*}\right)^{2} W}{\pi R^{3}}\left(\frac{B\left(x^{*}\right)}{L\left(x^{*}\right)} p^{*}\left(x^{*}\right)\right)^{\frac{1}{n}} \\
& \left(1-\frac{L\left(x^{*}\right)}{B\left(x^{*}\right)} \frac{N_{\mathrm{HB}}}{p^{*}\left(x^{*}\right)}\right)^{\frac{n+1}{n}}\left(n+1+n \frac{L\left(x^{*}\right)}{B\left(x^{*}\right)} \frac{N_{\mathrm{HB}}}{p^{*}\left(x^{*}\right)}\right)
\end{aligned}
$$

Hereafter we call $q_{z}{ }^{*}-1$ the deviation of outflow from uniformity. It becomes zero in the entire region of $x^{*}\left(0 \leq x^{*} \leq 1\right)$ when we achieve uniformity in the slot outflow. The relation between $q_{z}^{*}$ and $Q_{x}^{*}$ is

$$
q_{z}^{*}=-\frac{d Q_{x}^{*}}{d x^{*}}
$$

\subsection{Derivation of the Optimum Geometry of the Slot}

\subsubsection{Outline of the deriving procedure}

We derive the optimum geometry of the slot using the equations for both the cavity and the slot.

First, by making a Taylor expansion of the equation for the cavity, a third order equation for $p^{* 1}\left(x^{*}\right)$ is obtained, under the condition of outflow uniformity. Then, $p^{*}\left(x^{*}\right)$ is given by integrating $p^{* \prime}\left(x^{*}\right)$. The initial value $p^{*}(1)$ needed for the integration is given algebraically by solving the third order polynomial equation of $p^{*}\left(x^{*}\right)$ which is obtained from Taylor expansion of the equation of $q_{z}^{*}$ expressing the slot flow.

Finally, the optimum slot geometry $\left(B\left(x^{*}\right), L\left(x^{*}\right)\right)$ is given by solving the expressions for $q_{z}^{*}$ and $p^{*}\left(x^{*}\right)$ using the similar way of approximation as used above.

\subsubsection{Derivation of the pressure condition in the cavity} for attaining uniform slot outflow

In the following, $p^{*}\left(x^{*}\right)$ is derived, under the condition of the outflow uniformity. When the outflow uniformity is achieved, that is, $q_{z}{ }^{*}=1$, the following Eq. (16) is derived from Eq. (15).

$$
Q_{x}^{*}=1-x^{*}
$$

We now obtain the following equation from Eq. (16),

$$
\begin{aligned}
& \left(\frac{(n+1)(2 n+1)(3 n+1)\left(1-x^{*}\right)}{n}\right)^{n} \frac{\alpha\left(x^{*}\right)}{N_{\mathrm{HB}}} \\
& =\left(1-\alpha\left(x^{*}\right)\right)^{n+1} E\left(\alpha\left(x^{*}\right)\right) \\
& \alpha\left(x^{*}\right)=-\frac{2 W}{R} \frac{N_{\mathrm{HB}}}{p^{* 1}\left(x^{*}\right)}
\end{aligned}
$$

$$
E\left(\alpha\left(x^{*}\right)\right)=\left(2 n^{2} \alpha\left(x^{*}\right)^{2}+2 n(n+1) \alpha\left(x^{*}\right)+(n+1)(2 n+1)\right)^{n}
$$

Equation (17) shows that $p^{* 1}\left(x^{*}\right)$ is a function of $W, R, n, N_{\mathrm{HB}}$ and $x^{*}$, and not of the slot geometry parameters $B, L$ as shown by Eq. (18).

Here $\alpha\left(x^{*}\right)$ is a dimensionless parameter relevant to comparison of yield stress with pressure in the cavity $\left(0 \leq \alpha\left(x^{*}\right) \leq 1\right)$. The parameter $\alpha\left(x^{*}\right)$ is positive for fluids with yield stresses. It is obvious from Eq. (17) that the flow in the cavity stops at $x^{*}=1$, and then $\alpha(1)=1$.

We derive $\alpha\left(x^{*}\right)$ as follows:

By making a Taylor expansion about $\alpha\left(x^{*}\right)=1$ of Eq. (19), we have

$$
E\left(\alpha\left(x^{*}\right)\right)=((2 n+1)(3 n+1))^{n}\left(1+\sum_{k=1}^{\infty} b_{\alpha k}\left(\alpha\left(x^{*}\right)-1\right)^{k}\right)
$$

Here $b_{\alpha k}$ is the coefficients of each $k$ th term $(k \geq 1)$ for Taylor expansion at $\alpha\left(x^{*}\right)=1$. The term $b_{\alpha k}\left(\alpha\left(x^{*}\right)-1\right)^{k}$ is negligible compared with unity if $k \geq 2$. We ignore higher-order terms in comparison to linear terms in Eq. (20), and substitute it for Eq. (17). We then obtain the following expression.

$$
\begin{aligned}
& \left(\frac{(n+1)\left(1-x^{*}\right)}{n}\right)^{n} \frac{\alpha\left(x^{*}\right)}{N_{\mathrm{HB}}} \\
& =\left(1-\alpha\left(x^{*}\right)\right)^{n+1}\left(1-\frac{2 n^{2}}{2 n+1}\left(1-\alpha\left(x^{*}\right)\right)\right)
\end{aligned}
$$

A Taylor expansion can be made about $\alpha\left(x^{*}\right)=1 / 2$ in the right-hand side of Eq. (21), in view of the factor of $1-\alpha\left(x^{*}\right)$. The result is

$$
\begin{aligned}
& \left(1-\alpha\left(x^{*}\right)\right)^{n+1}\left(1-\frac{2 n^{2}}{2 n+1}\left(1-\alpha\left(x^{*}\right)\right)\right) \\
& =2^{-n-1} \frac{-n^{2}+2 n+1}{2 n+1}\left(1+\sum_{k=1}^{\infty} c_{\alpha k}\left(\alpha\left(x^{*}\right)-\frac{1}{2}\right)^{k}\right)
\end{aligned}
$$


where $c_{\alpha k}$ is the coefficients of the $k$ th term $(k \geq 1)$ in the Taylor expansion about $\alpha\left(x^{*}\right)=1 / 2$. The $c_{\alpha k}\left(\alpha\left(x^{*}\right)-1 / 2\right)^{k}$ term is negligible compared with unity for $k \geq 4$. Therefore, higherorder terms than cubic are ignored in Eq. (22), and the result is substituted using Eq. (17). The result is

$$
\sum_{k=0}^{3} a_{\alpha k} \alpha\left(x^{*}\right)^{k}=0
$$

where $a_{\alpha k}$ is given as follows.

$$
\begin{aligned}
a_{\alpha 0}= & \frac{-n^{5}-4 n^{4}-10 n^{3}-5 n^{2}+32 n+12}{8 n(n+1)\left(n^{3}+n+1\right)}, \\
a_{\alpha 1}= & \frac{3\left(n^{5}+3 n^{4}+5 n^{3}-7 n^{2}-2\right)}{4 n(n+1)\left(n^{3}+n+1\right)} \\
& -\frac{3 \cdot 2^{n-1}(2 n+1)(n+1)^{n-1}\left(1-x^{*}\right)^{n}}{n^{n+1}\left(n^{3}+n+1\right) N_{\mathrm{HB}}}, \\
a_{\alpha 2}= & -\frac{3\left(n^{2}+n+1\right)}{2\left(n^{3}+n+1\right)}, \quad a_{\alpha 3}=1
\end{aligned}
$$

Equation (23) is certainly able to be solved and to give at least one real root of algebraic form, since we have cubic equations for $\alpha\left(x^{*}\right)$ with real coefficients. Next, $p^{*}\left(x^{*}\right)$ is given in terms of $\alpha\left(x^{*}\right)$ and Eq. (18) as

$$
p^{*}\left(x^{*}\right)=p^{*}(1)+\int_{x^{*}}^{1} \frac{2 W}{R} \frac{N_{\mathrm{HB}}}{\alpha\left(x^{*}\right)} d x^{*}
$$

$p^{*}(1)$ in Eq. (25) is not known at the present stage.

Here $p^{*}(1)$ is derived as follows: When outflow uniformity is achieved, that is, $q_{z}{ }^{*}=1$, Eq. (14) becomes

$$
\left(\frac{(n+1)(2 n+1)}{2 n} \frac{\pi R^{3}}{B\left(x^{*}\right)^{2} W}\right)^{n} \frac{\beta\left(x^{*}\right)}{N_{\mathrm{HB}}}=\left(1-\beta\left(x^{*}\right)\right)^{n+1}\left(n+1+n \beta\left(x^{*}\right)\right)^{n}
$$

where $\beta\left(x^{*}\right)$ is defined as

$$
\beta\left(x^{*}\right)=\frac{L\left(x^{*}\right)}{B\left(x^{*}\right)} \cdot \frac{N_{\mathrm{HB}}}{p^{*}\left(x^{*}\right)}
$$

$\beta\left(x^{*}\right)$, which is similar to $\alpha\left(x^{*}\right)$, is a dimensionless parameter relevant in comparing yield stress with pressure in the slot $\left(0 \leq \beta\left(x^{*}\right) \leq 1\right)$. It is positive for the flow of fluids having yield stresses.

The flow in the slot stops when $\beta\left(x^{*}\right)=1$. However the value of $\beta\left(x^{*}\right)$ is nearer zero in practice. By Taylor expansion about $\beta\left(x^{*}\right)=0$ in the right-hand side of Eq. (26), we have

$$
\left(1-\beta\left(x^{*}\right)\right)^{n+1}\left(n+1+n \beta\left(x^{*}\right)\right)^{n}=(n+1)^{n}\left(1+\sum_{k=1}^{\infty} c_{\beta k} \beta\left(x^{*}\right)^{k}\right)
$$

where $c_{\beta k}$ is the coefficients of each $k$ th term $(k \geq 1)$ for Taylor expansion at $\beta\left(x^{*}\right)=0$. The $c_{\beta k} \beta\left(x^{*}\right)^{k}$ term is far smaller than unity for $k \geq 4$. Therefore, terms of higher order than cubic are ignored in Eq. (28), and applied in Eq. (26). The result is

$$
\sum_{k=0}^{3} a_{\beta k} \beta\left(x^{*}\right)^{k}=0
$$

where $a_{\beta k}$ is given as follows.

$$
\begin{aligned}
& \left.a_{\beta 0}=1, \quad a_{\beta 1}=-\frac{2 n+1}{n+1}-\left(\frac{2 n+1}{2 n} \cdot \frac{\pi R^{3}}{B\left(x^{*}\right)^{2} W}\right)\right)^{n} \frac{1}{N_{\mathrm{HB}}}, \\
& a_{\beta 2}=-\frac{n(n-1)(2 n+1)}{2(n+1)^{2}}, \quad a_{\beta 3}=\frac{n(2 n+1)\left(6 n^{2}+n+1\right)}{6(n+1)^{3}}
\end{aligned}
$$

Being a cubic equation for $\beta\left(x^{*}\right)$ with real coefficients, Eq. (29) can be solved and has at least one real root.

Now $p^{*}\left(x^{*}\right)$ is given by the root of $\beta\left(x^{*}\right)$ and Eq. (27), and $p^{*}(1)$ is determined from the fact that $B\left(x^{*}\right)=B$ and $L\left(x^{*}\right)=L$ at $x^{*}=1$. Therefore $p^{*}\left(x^{*}\right)$ is given by solving Eq. (25) as follows.

$$
p^{*}\left(x^{*}\right)=\frac{L}{B} \cdot \frac{N_{\mathrm{HB}}}{\beta(1)}+\int_{x^{*}}^{1} \frac{2 W}{R} \frac{N_{\mathrm{HB}}}{\alpha\left(x^{*}\right)} d x^{*}
$$

Solving Eq. (31) involves numerical calculation, because the integral cannot be expressed in algebraic form. However, it is easy to obtain $p^{*}\left(x^{*}\right)$ accurately in practice.

\subsubsection{Derivation of the optimum slot geometry giving outflow uniformity}

$B\left(x^{*}\right)$ or $L\left(x^{*}\right)$ for the optimum slot geometry can be derived by solving Eq. (14) for $p^{*}\left(x^{*}\right)$ under the condition that $q_{z}^{*}$ is unity. Although we can vary both $B\left(x^{*}\right)$ and $L\left(x^{*}\right)$, we show only the case in which $B\left(x^{*}\right)$ varies with location $x^{*}$ (then $L\left(x^{*}\right)$ takes a constant value $L$ ), because it is much easier to vary $B\left(x^{*}\right)$ in practical machining processes than $L\left(x^{*}\right)$.

The following Eq. (32) is given by Eq. (14) with $q_{z}^{*}$ set to unity.

$$
\begin{aligned}
& \left(\frac{2 n+1}{2 n} \cdot \frac{\pi R^{3}}{B^{2} W}\right)^{n} \frac{\beta_{1}\left(x^{*}\right)}{N_{\mathrm{HB}}}=\left(\frac{1}{n+1}\right)^{n} \\
& \left(B^{*}\left(x^{*}\right)-\beta_{1}\left(x^{*}\right)\right)^{n+1}\left((n+1) B^{*}\left(x^{*}\right)+n \beta_{1}\left(x^{*}\right)\right)^{n}
\end{aligned}
$$

where $B^{*}\left(x^{*}\right)$ and $\beta_{1}\left(x^{*}\right)$ are defined as

$$
\begin{aligned}
& B^{*}\left(x^{*}\right)=\frac{B\left(x^{*}\right)}{B} \\
& \beta_{1}\left(x^{*}\right)=\frac{L}{B} \cdot \frac{N_{\mathrm{HB}}}{p^{*}\left(x^{*}\right)}
\end{aligned}
$$


$B^{*}\left(x^{*}\right)$ is a normalized parameter expressing the variation of the $y$-directional gap with location $x^{*}$, and is unity at $x^{*}=1$. $B^{*}\left(x^{*}\right)$ is at least $2 / 3$ and is less than unity in practical manufacturing processes of slot dies.

We now make a Taylor expansion about $B^{*}\left(x^{*}\right)=1$ of the factor of $\left(B^{*}\left(x^{*}\right)-\beta_{1}\left(x^{*}\right)\right)^{n+1}$ and the factor of $\left((\mathrm{n}+1) B^{*}\left(x^{*}\right)+n \beta_{1}\left(x^{*}\right)\right)^{n}$ in the right-hand side of Eq. (32).

$$
\left(B^{*}\left(x^{*}\right)-\beta_{1}\left(x^{*}\right)\right)^{n+1}=\left(1-\beta_{1}\left(x^{*}\right)\right)^{n+1}\left(1+\sum_{k=1}^{\infty} a_{B k}\left(B^{*}\left(x^{*}\right)-1\right)^{k}\right)
$$

$$
\begin{aligned}
& \left((n+1) B^{*}\left(x^{*}\right)+n \beta_{1}\left(x^{*}\right)\right)^{n} \\
& \quad=\left(1+n+n \beta_{1}\left(x^{*}\right)\right)^{n}\left(1+\sum_{k=1}^{\infty} b_{B k}\left(B^{*}\left(x^{*}\right)-1\right)^{k}\right)
\end{aligned}
$$

where $a_{B k}$ and $b_{B k}$ denote the coefficients of each $k$ th terms $(k \geq 1)$ for Taylor expansion at $B^{*}\left(x^{*}\right)=1$.

The term $a_{B k}\left(B^{*}\left(x^{*}\right)-1\right)^{k}$ in Eq. (35) is negligible compared with unity for $k \geq 3$. The term $b_{B k}\left(B^{*}\left(x^{*}\right)-1\right)^{k}$ in Eq. (36) is negligible compared with unity for $k \geq 2$.

Therefore, terms higher than quadratic are ignored in Eq. (35) and higher than linear in Eq. (36), and the truncations are substituted in Eq. (32).

Finally, ignoring terms of higher order than cubic in Eq. (32), the following expression is derived:

$$
\begin{aligned}
& \sum_{k=0}^{3} c_{B k}\left(B^{*}\left(x^{*}\right)-1\right)^{k}=0 \\
& c_{B 0}=\frac{2\left(1-\beta_{1}\left(x^{*}\right)\right)^{2}\left(1+n+n \beta_{1}\left(x^{*}\right)\right)}{n^{2}(n+1)^{2}} \\
& \quad\left(1-\left(\frac{(n+1)(2 n+1)}{2 n\left(1+n+n \beta_{1}\left(x^{*}\right)\right)} \cdot \frac{\pi R^{3}}{B^{2} W}\right)^{n} \frac{\beta_{1}\left(x^{*}\right)}{N_{\mathrm{HB}}\left(1-\beta_{1}\left(x^{*}\right)\right)^{n+1}}\right), \\
& c_{B 1}=\frac{2(2 n+1)\left(1-\beta_{1}\left(x^{*}\right)\right)}{n^{2}(n+1)}, \\
& c_{B 2}=-\frac{(n+2) \beta_{1}\left(x^{*}\right)-3 n-3}{n(n+1)}, \\
& c_{B 3}=1
\end{aligned}
$$

Equation (37) can be solved algebraically giving at least one real root because it is a cubic equation for $B^{*}\left(x^{*}\right)$ having real coefficients. The optimum slot geometry $B\left(x^{*}\right)$ is given by solving $B^{*}\left(x^{*}\right)$ together with Eq. (33).

\section{RESULTS AND DISCUSSION: EXPERIMENT AND CALCULATION}

\subsection{Materials}

Three kinds of test fluids were used: a Herschel-Bulkley fluid (Sample 1), a Bingham fluid ${ }^{20)}$ (Sample 2), and a Newtonian fluid ${ }^{20)}$ (Sample 3).

Sample 1 consisted of silica particle $(\phi 3.0 \mu \mathrm{m}$, $\left(\rho=8.0 \times 10^{1} \mathrm{~kg} / \mathrm{m}^{3}\right)$ manufactured by Tokai Chemical Industry Co., Ltd., silicone oil $\left(\mu_{0}=1.00 \times 10^{-2} \mathrm{~Pa} \bullet \mathrm{s}\right)$ by GE Toshiba Silicone Co., Ltd. and solution of acetate $(5 \mathrm{wt} \%)$ mixed in a weight ratio of 1/16.2/0.086.

Sample 2 consisted of nylon particle $\left(\phi 4.1 \mu \mathrm{m},\left(\rho=1.02 \times 10^{3} \mathrm{~kg} / \mathrm{m}^{3}\right)\right.$ manufactured by Toray Industries, Inc., silicone oil $\left(\mu_{0}=1.00 \times 10^{-1} \mathrm{~Pa} \bullet \mathrm{s}\right)$ by GE Toshiba Silicone Co., Ltd. and solution of acetate $(5 \mathrm{wt} \%)$ mixed in a weight ratio of $1 / 3.88 / 0.125$.

Sample 3 was made by mixing low viscosity $\left(\mu_{0}=1.00 \times 10^{-1}\right.$ $\mathrm{Pa} \bullet \mathrm{s})$ silicone oil $(65.5 \mathrm{wt} \%)$ and high viscosity $\left(\mu_{0}=1.00 \mathrm{~Pa} \bullet \mathrm{s}\right)$ oil(34.5 wt $\%)$, both manufactured by GE Toshiba Silicone Co., Ltd.

Figure 2 and Table I show the rheological properties of test fluids measured by a stress controlled Physica MCR301 rheometer manufactured by Anton Paar GmbH.

\subsection{Apparatus}

The system used in the present experiment mainly consisted of a fluid tank, a power department and a slot die. To

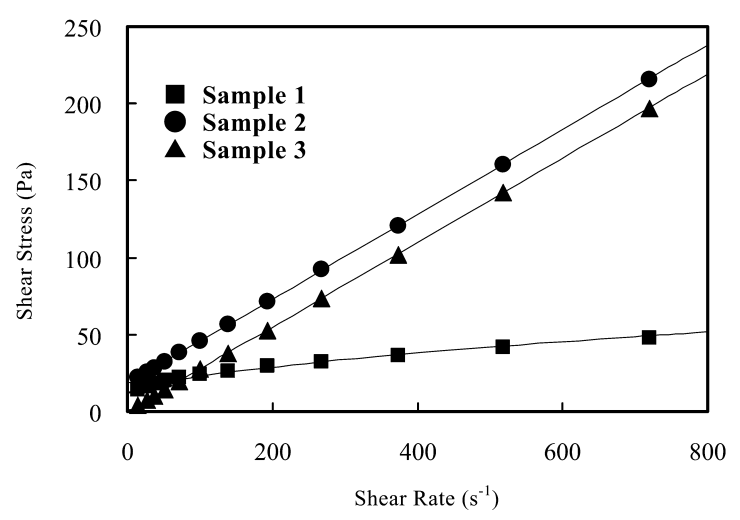

Fig. 2. Rheological properties of test fluids.

Table I. Rheological properties of test fluids measured at $25^{\circ} \mathrm{C}$.

\begin{tabular}{|c|c|c|c|c|}
\hline Sample No. & $\mu_{0}(\mathrm{~Pa} \cdot \mathrm{s})$ & $\tau_{0}(\mathrm{~Pa})$ & $n(-)$ & Fluid Model \\
\hline 1 & 0.738 & 11.1 & 0.60 & Herschel-Bulkley \\
\hline 2 & 0.275 & 18.0 & 1.0 & Bingham \\
\hline 3 & 0.274 & 0.0 & 1.0 & Newtonian \\
\hline
\end{tabular}


overcome resistance due to stress in the fluids, a plunger pump was used.

The dimensions inside the slot die used are : $W=0.140 \mathrm{~m}$, $R=3.06 \times 10^{-3} \mathrm{~m}, L=20.0 \times 10^{-3} \mathrm{~m}, B=0.15 \times 10^{-3} \mathrm{~m}$. For all tests reported here, $L\left(x^{*}\right)$ was set to be a constant $L$. Also the geometry setting parameters $(W, R, L, B)$ were kept constant.

A segmented device was used for collecting the liquid issuing from each position of the slot exit for a time, so as to measure the distribution of flow rates at the slot exit and to determine the outflow deviation along the $x$-direction. It follows from Eqs. (17), (18), (19), (26), (27) and (31) that both $p^{*}\left(x^{*}\right)$ and $B\left(x^{*}\right)$ are functions of only $n$ and $N_{\mathrm{HB}}$ if the geometry setting parameters $(W, R, L, B)$ are constant.

If $n$ is fixed by the properties of the test fluids, $N_{\mathrm{HB}}$ can be varied arbitrarily by controlling the value of $Q_{0} . N_{\mathrm{HB}}=5.0$ was chosen as a standard flow condition and $Q_{0}=5.65 \times 10^{-7} \mathrm{~m}^{3} / \mathrm{s}$ for Sample 1 (Herschel-Bulkley fluid). This value of $Q_{0}$ is also used in the case of Newtonian fluid (Sample 3) in which $N_{\mathrm{HB}}=0$. For Sample 2 (Bingham fluid), $Q_{0}=1.17 \times 10^{-6} \mathrm{~m}^{3} / \mathrm{s}$, corresponding to $N_{\mathrm{HB}}=5.0$.

Figure 3 shows the width-wise flow uniformity in a die having constant slot geometry $\left(B\left(x^{*}\right)=B, L\left(x^{*}\right)=L\right)$ using Samples 1, 2 and 3. It can be seen that the Herschel-Bulkley fluid shows greater deviation than the Bingham or the Newtonian fluid. A possible reason is that the HerschelBulkley fluid shows greater resistance than the other fluids due to the yield stress towards the end of the cavity, which causes a decrease of the flow rate of the fluid flowing into the slot. Furthermore, the Herschel-Bulkley fluid has less resistance due to shear thinning towards the inlet of the cavity, which causes an increase of the slot flow rate.

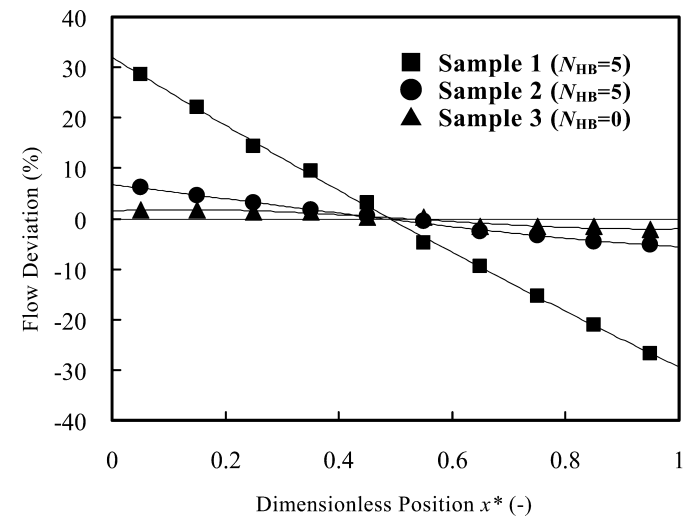

Fig. 3. Uniformity of the widthwise experimental flow in a die having the constant slot geometry.

\subsection{Comparison with Algebraic Analyses in Previous Studies}

To compare the present analysis with previous algebraic studies it is necessary to examine the influences of shear thinning and yield stress separately, since it is difficult to find previous studies dealing with both influences at once.

Next, the present analytical method is compared with Gutoff's method for investigating the effect of shear thinning, as exhibited by the power-law model. ${ }^{8)}$

Gutoff gave the following equations for the optimum geometry.

$$
\begin{aligned}
& B\left(x^{*}\right)=\left(\frac{2 n+1}{2 n} \cdot \frac{\pi R^{3}}{W}\right)^{\frac{n}{2 n+1}}\left(\frac{L}{p^{*}\left(x^{*}\right)}\right)^{\frac{1}{2 n+1}} \\
& p^{*}\left(x^{*}\right)=\frac{2}{n+1}\left(\frac{3 n+1}{n}\right)^{n} \frac{W}{R}\left(1-x^{*}\right)^{n+1}+\left(\frac{2 n+1}{2 n} \cdot \frac{\pi R^{3}}{B^{2} W}\right)^{n} \frac{L}{B}
\end{aligned}
$$

Figure 4 shows the optimum slot geometries $B\left(x^{*}\right)$ for power-law fluids calculated by the two methods. That the results agree closely with each other is obvious.

Similarly, we compare the present method with the method described in our previous report which gives the optimum slot geometry for Bingham fluid. ${ }^{20)}$

Figure 5 shows the result of both methods for Bingham fluid, varying only $N_{\mathrm{HB}}$. Here quite good agreement between the present and previous methods is evident.

Figures 6 and 7 show the difference between the results of the present method and the previous method for each fluid model. The discrepancy is largest for Newtonian fluid and reduces as $n$ and $N_{\mathrm{HB}}$ increase. The difference is within the allowable range, according to the accuracy of the machining process.

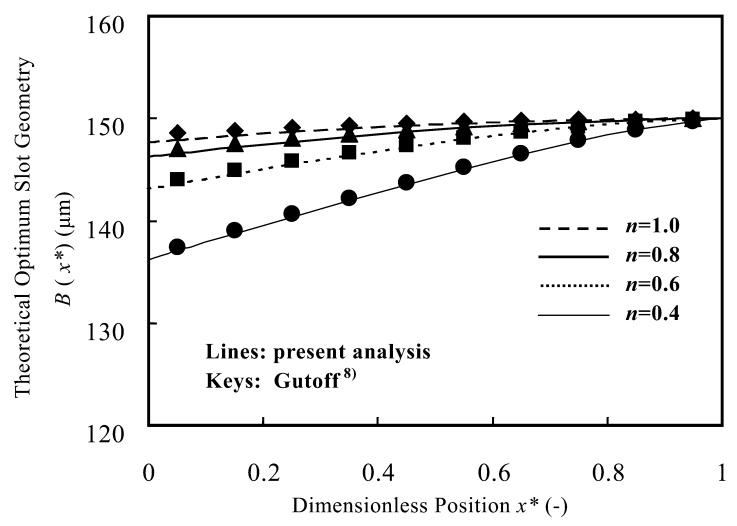

Fig. 4. Comparison of the present analysis with the previous one in predictions of the optimum slot geometry for power-law fluids. 


\subsection{Comparison between Analytical and Experimental Results}

As seen above, the slot geometry $B\left(x^{*}\right)$ is a function of only $n$ and $N_{\mathrm{HB}}$ when the geometry setting parameters are given in advance. Figures 8 and 9 show the results for optimum slot $B\left(x^{*}\right)$ calculated by varying $n$ and $N_{\mathrm{HB}}$, respectively.

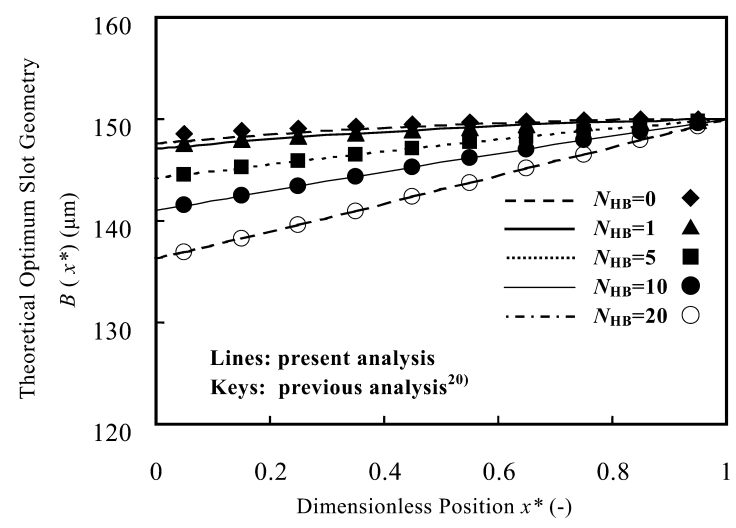

Fig. 5. Comparison of the present analysis with the previous one in predictions of the optimum slot geometry for Bingham fluids.

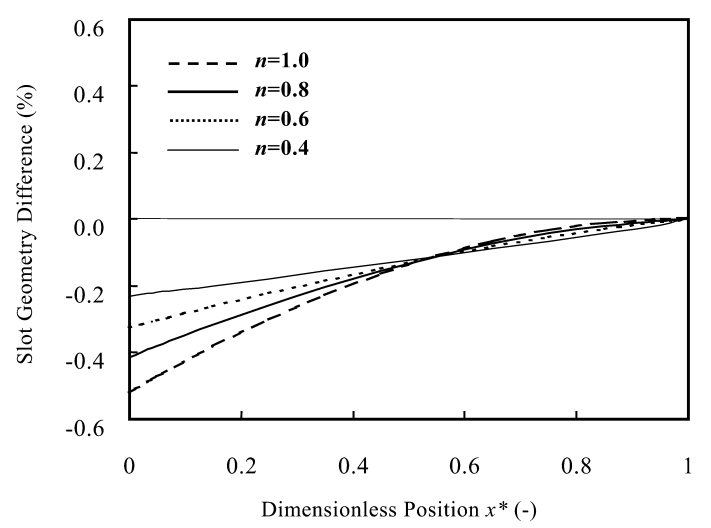

Fig. 6. Difference between the present analysis and the previous one in predictions of the optimum slot geometry for power-law fluids.

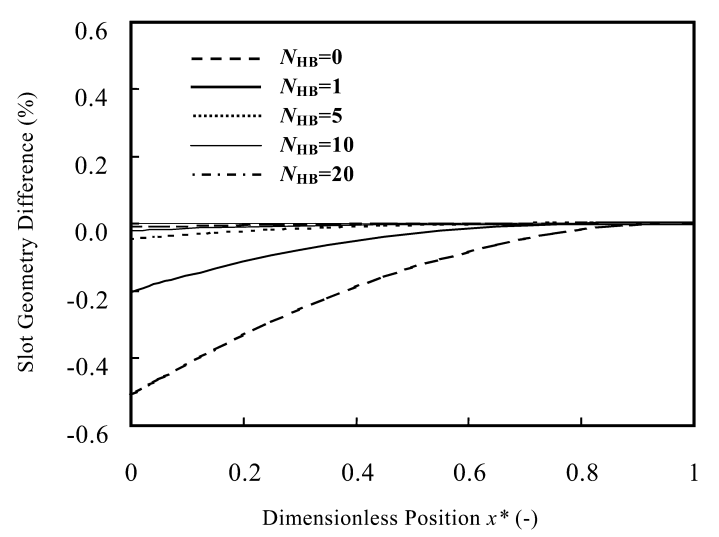

Fig. 7. Difference between the present analysis and the previous one in predictions of the optimum slot geometry for Bingham fluids.
Figure 8 shows that the lower is the value of $n$, the greater the variation in slot geometry, as in Fig. 4. Figure 9 shows that greater variation is needed for larger values of $N_{\mathrm{HB}}$, as in Fig.5.

Figure 10 shows the experimental results of outflow deviation using Sample 1 in a die having optimum slot geometry. We find that outflow uniformity can be achieved in

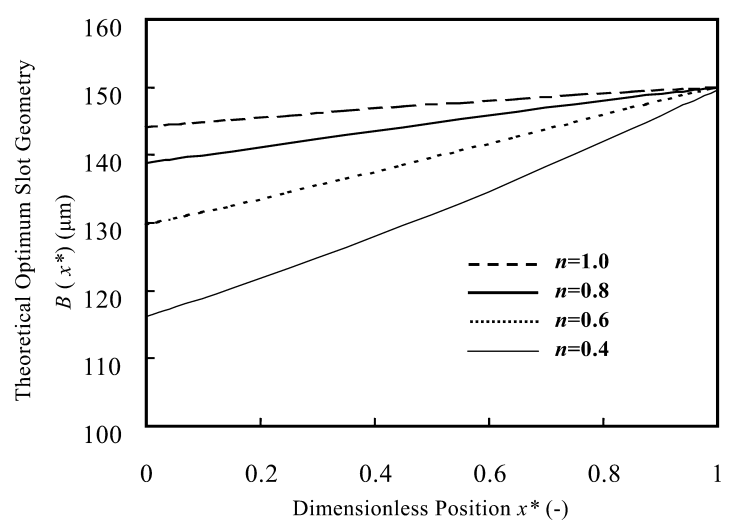

Fig. 8. Theoretical predictions of the optimum slot geometry for various n. $\left(N_{\mathrm{HB}}=5.0\right)$

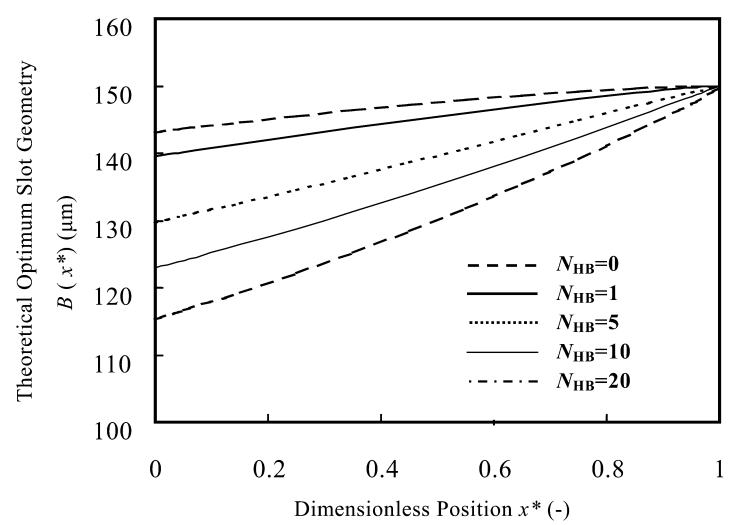

Fig. 9. Theoretical predictions of the optimum slot geometry for various $N_{\mathrm{HB}} \cdot(n=0.6)$

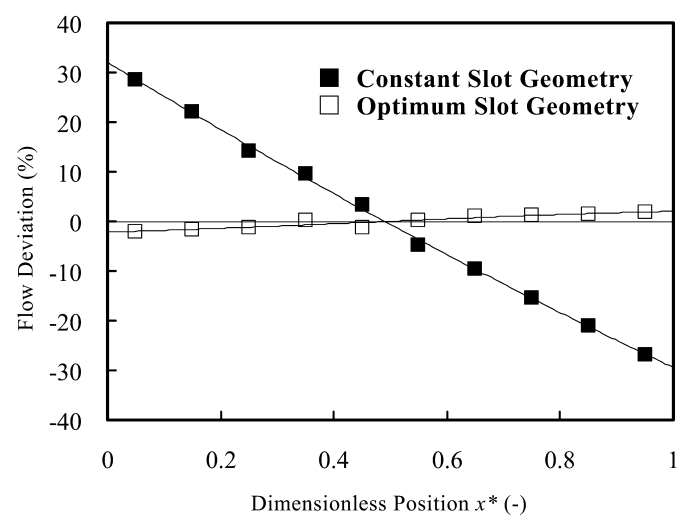

Fig. 10. Uniformity of the widthwise experimental flow in a die having the optimum slot geometry. 
practice by using the die having optimum slot geometry for Herschel-Bulkley fluid.

\section{CONCLUSION}

The present study has clarified the effect of shear thinning and yield stress on flow distributions, and proposes a method for designing the optimum geometry inside the die in the case of Herschel-Bulkley fluid. Furthermore, it has been shown that the proposed method is efficient and easy to use, comparing it with the results of the experiments carried out in this study and with previous analytical studies using the power-law fluid model and Bingham fluid model.

Several methods for designing slot dies have been developed so far, for the power-law model, Ellis model, Carreau-Yasuda model, etc., but few methods have been found for a fluid model exhibiting both non-Newtonian viscosity and yield stress at once.

This study proposes a useful analytical method for the Herschel-Bulkley model, which exhibits both non-Newtonian viscosity and yield stress.

\section{REFERENCES}

1) Tsuda $T$, Hasegawa $T$, Narumi $T$, Journal of the Japan Society of Mechanical Engineers Series B, 67, 2174 (2001).

2) Iida Y, Coating Gijutsu, 37 (1999), Technical Information Institute Co., Ltd.
3) Kigasawa T, Nojo K, Coating, 157 (1999), Converting Technical Institute.

4) Durst F, Lange U, Raszillier H, Chem Engng Sci, 49, 161 (1994).

5) Spasojević D, Irvine TF, Afgan N, International Journal of Multiphase Flow, 1, 607 (1974).

6) Yano S, Otsuka K, Kawai A, Hagiwara T, Sawaguchi T, Nihon Reoroji Gakkaishi (J Soc Rheol, Jpn), 33, 23 (2005).

7) Narumi T, See H, Oumi Y, Satou F, Hasegawa T, Nihon Reoroji Gakkaishi (J Soc Rheol, Jpn), 33, 29 (2005).

8) Gutoff EB, Journal of Imaging Science Technology, 37, 615 (1993).

9) Smith DE, Wang Q, Polym Engng Sci, 45, 953 (2005).

10) Wang Y, Polym Engng Sci, 31, 204 (1991).

11) Lee KY, Liu TJ, Polym Engng Sci, 29, 1066 (1989).

12) Lee KY, Wen SH, Liu TJ, Polym Engng Sci, 30, 1220 (1990)

13) Weinstein SJ, Ruschak KJ, AIChE Journal, 42, 1501 (1996).

14) Weinstein SJ, Ruschak KJ, AIChE Journal, 42, 2401 (1996).

15) Yuan SL, Polym Engng Sci, 35, 577 (1995).

16) Kamişli F, International Journal of Engineering Science, 41, 1059 (2003).

17) Tsuda $T$, Hasegawa $T$, Narumi $T$, Nihon Reoroji Gakkaishi (J Soc Rheol, Jpn), 30, 133 (2002).

18) Papanastasiou TC, Journal of Rheology, 31, 385 (1987).

19) Zhu H, Kim YD, Kee DD, Journal of Non-Newtonian Fluid Mechanics, 129, 177 (2005).

20) Nagashima $M$, Hasegawa $T$, Narumi $T$, Nihon Reoroji Gakkaishi (J Soc Rheol, Jpn), 34, 91 (2006).

21) Leonard WK, Polym Engng Sci, 25, 570 (1985).

22) Tomita Y, Rheology, 316 (1975), Corona Publishing Co., Ltd. 